\title{
Crystal structure of the IL-15-IL-15R $\alpha$ complex, a cytokine-receptor unit presented in trans
}

\author{
Mami Chirifu ${ }^{1,2}$, Chiharu Hayashi ${ }^{1,2}$, Teruya Nakamura ${ }^{1}$, Sachiko Toma ${ }^{1,3}$, Tsuyoshi Shuto ${ }^{1}$, Hirofumi Kai ${ }^{1}$, \\ Yuriko Yamagata $^{1}$, Simon J Davis ${ }^{4}$ \& Shinji Ikemizu ${ }^{1,2}$
}

Interleukin 15 (IL-15) and IL-2, which promote the survival of memory CD8 ${ }^{+} \mathrm{T}$ cells and regulatory T cells, respectively, bind receptor complexes that share $\beta$ - and $\gamma$-signaling subunits. Receptor specificity is provided by unique, nonsignaling $\alpha$-subunits. Whereas IL-2 receptor- $\alpha$ (IL-2R $\alpha$ ) is expressed together in cis with the $\beta$ - and $\gamma$-subunits on T cells and B cells, IL-15R $\alpha$ is expressed in trans on antigen-presenting cells. Here we present a 1.85-Å crystal structure of the human IL-15-IL-15R $\alpha$ complex. The structure provides insight into the molecular basis of the specificity of cytokine recognition and emphasizes the importance of water in generating this very high-affinity complex. Despite very low IL-15-IL-2 sequence homology and distinct receptor architecture, the topologies of the IL-15-IL-15R $\alpha$ and IL-2-IL-2R $\alpha$ complexes are very similar. Our data raise the possibility that IL-2, like IL-15, might be capable of being presented in trans in the context of its unique receptor $\alpha$-chain.

Cytokines of the common $\gamma$-chain $(\gamma c)$ family, which include interleukin 2 (IL-2), IL-4, IL-7, IL-9, IL-15 and IL-21, are small soluble proteins, all of which bind receptors sharing the common signaling subunit $\gamma c^{1}$. These cytokines have overlapping functions and affect the activity and functions of lymphocytes at various stages of development, but their main function is the control of $\mathrm{T}$ cell survival and proliferation ${ }^{2}$. IL-15, along with IL-7, controls memory T cell homeostasis ${ }^{3,4}$.

IL-15 was discovered in 14- to 15-kilodalton cytokine preparations whose cell-binding activity was blocked by antibodies specific for IL-2 receptor- $\beta$ (IL-2R $\beta)^{5,6}$. Initial comparisons failed to show any nucleotide or protein sequence similarity between IL-15 and IL-2 (ref. 6), but subsequent secondary structure predictions indicated that IL-15, like IL-2, belongs to the family of four helix-bundle cytokines ${ }^{7}$. A unique subunit, IL-15R $\alpha$, was subsequently shown to be required, along with the signaling subunits IL-2R $\beta$ and IL- $2 \mathrm{R} \gamma^{8}$, for formation of the high affinity IL-15R ${ }^{9}$. Only IL- 15 and IL-2 share IL-2R $\beta$; IL-2R $\gamma$ is shared with all other $\gamma$ c family cytokines ${ }^{1-4}$. The affinity of IL-15R $\alpha$ for IL-15 $(38 \mathrm{pM})^{10}$, is much higher than that of the IL-2-IL2R $\alpha$ interaction $(28.1 \mathrm{nM})^{11}$. IL- $15 \mathrm{R} \alpha$ shows structural similarity with the IL- $2 \mathrm{R} \alpha$ subunit of IL-2R (also called CD25) insofar as both $\alpha$-chains contain a 'sushi' domain of the type found in complement-related and other proteins, a region rich in threonine and proline residues, and a short cytoplasmic domain 9 . IL15RA and IL2RA are very closely linked on human chromosome 10, suggesting duplication of an ancient precursor of both genes ${ }^{12}$. Because these two cytokines share signaling receptor subunits, IL-15-induced membrane-proximal signaling by means of the Jak and STAT signaling proteins may be identical to that induced by IL-2 (refs 1,13).

Perhaps the most salient property of IL-15 is that it is presented in trans $^{1,4,13}$. IL-15 is mostly undetectable in soluble form and its mRNA is only very weakly expressed by T cells ${ }^{14}$. IL-15 is constitutively expressed by epithelial cells, fibroblasts, monocytes and dendritic cells but must be coexpressed with IL-15R $\alpha$ to be functional; mixed bone marrow chimeras derived from IL-15- and IL-15R $\alpha$-deficient mice fail to complement each other in IL- $15 \mathrm{R} \alpha$ deficient hosts ${ }^{15}$. The idea that IL-15 is trans-presented by IL-15R $\alpha$ expressing myeloid lineage cells to $\mathrm{T}$ cells expressing IL-2R $\beta$ and $\gamma \mathrm{c}^{16}$ arose after it was shown that wild-type $\mathrm{CD} 8^{+} \mathrm{T}$ cells transferred into IL-15R $\alpha$-deficient mice do not proliferate, whereas IL-15R $\alpha$-deficient $\mathrm{CD}^{+} \mathrm{T}$ cells proliferate rapidly in wild-type hosts ${ }^{17}$. Thus, in contrast to the apparent in cis autocrine signaling triggered by IL-2, IL-15-dependent signaling is probably influenced by cell-cell contact.

Despite sharing receptor-signaling subunits, IL-15 and IL-2 exert distinct effects on $\mathrm{T}$ cell homeostasis, emphasizing the importance of signaling intensity, context and timing on signaling outcomes. The proliferation of memory-type $\mathrm{CD}^{+} \mathrm{T}$ cells, for example, is stimulated by IL-15 and is inhibited by IL-2 (ref. 18). Similarly, the development and survival of regulatory T cells is highly dependent on IL-2 but is independent of IL-15 (ref. 19). In addition, whereas IL-2 promotes the elimination of activated $\mathrm{T}$ cells ${ }^{20}$, IL-15 suppresses apoptosis ${ }^{21}$. IL-2-deficient mice are profoundly deficient in regulatory $\mathrm{T}$ cells ${ }^{22-24}$, and IL-15- and IL-15R $\alpha$-deficient mice have notable and selective

\footnotetext{
${ }^{1}$ Graduate School of Pharmaceutical Sciences and ${ }^{2} 21$ st Century Center of Excellence Project, Kumamoto University, 5-1 Oe-honmachi, Kumamoto 862-0973, Japan. ${ }^{3}$ Graduate School of Sciences and Pharmaceutical Sciences, The University of Tokyo, 7-3-1 Hongo Bunkyo-ku, Tokyo 113-0033, Japan. ${ }^{4}$ Nuffield Department of Clinical Medicine, and Medical Research Council Human Immunology Unit, The University of Oxford, The Weatherall Institute of Molecular Medicine, Headington, Oxford, OX3 9DS, UK. Correspondence should be addressed to S.I. (ikemizu@gpo.kumamoto-u.ac.jp).
} 

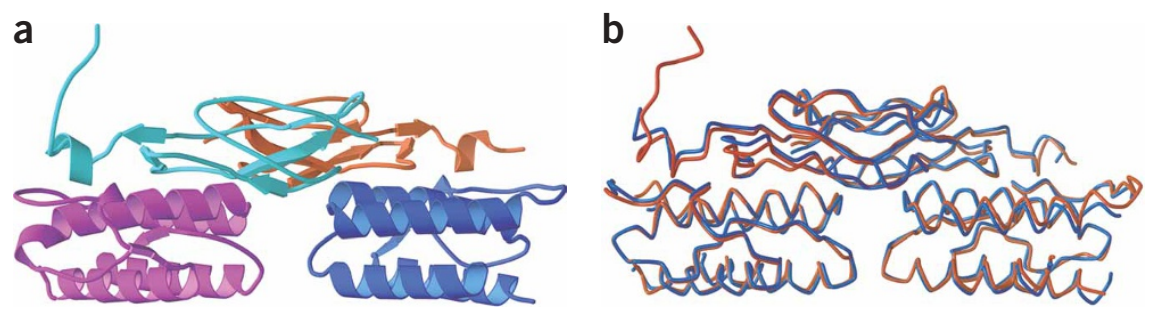

음

Figure 1 Structure of the human IL-15-IL-15R $\alpha$ complex. (a) Two IL-15-IL-15R $\alpha$ complexes in the asymmetric unit of the P ${ }_{1} 2{ }_{1} 2$ crystal form. One IL-15 molecule (magenta) forms a complex with one IL-15R $\alpha$ molecule (cyan), which then associates with another complex of IL-15 (blue) and IL-15R $\alpha$ (red). These two complexes have a noncrystallographic twofold relationship. (b) Superposition of dimers of IL-15-IL15R $\alpha$ complexes from the P $2{ }_{1}{ }_{2} 2$ (red) and P $2{ }_{1} 2_{1} 2_{1}$ (blue) crystals.

Table 1 Data collection and refinement statistics of the IL-15-IL-15R $\alpha$ complex, forming a rod-like dimer (Fig. 1a). A similar dimer formed in the $\mathrm{P} 2{ }_{1} 2_{1} 2_{1}$ lattice (r.m.s. deviation of $0.90 \AA$ for 370 residues; Fig. 1b), which contains eight copies of the IL-15-IL$15 \mathrm{R} \alpha$ complex. In the final models derived from the $\mathrm{P} 2{ }_{1} 2_{1} 2$ and $\mathrm{P} 2{ }_{1} 2_{1} 2_{1}$ crystals, $90.4 \%$ and $93.4 \%$ of the residues were in the most-favored regions of the Ramachandran plot $^{34}$, respectively, and none were in disallowed regions (data collection and refinement statistics, Table 1; representative electron density, Supplementary Fig. 1 online). reductions in the numbers of memory phenotype $\mathrm{CD}^{+} \mathrm{T}$ cells, natural killer T cells and intraepithelial lymphocytes ${ }^{25,26}$. It is therefore becoming accepted that IL-2 is central to the maintenance of peripheral tolerance, whereas IL-15 sustains high-avidity $\mathrm{T}$ cell responses to pathogens by enhancing $\mathrm{CD}^{+}$memory $\mathrm{T}$ cell survival. This view has prompted a switch in emphasis from IL-2 to IL-15 in the context of cancer immunotherapy and vaccine development ${ }^{1}$. Forced expression of IL-15R $\alpha$ in tumor cells prevents tumor progression ${ }^{27}$ and coexpression of IL-15 promotes prolonged immunity ${ }^{28}$. The crystal structures of IL-2 in complex with IL-2R $\alpha^{29}$ and with IL-2R $\alpha$, IL-2R $\beta$ and $\gamma c^{30,31}$ have been solved, as has a nuclear magnetic resonance (NMR)-based structure of IL-15R $\alpha$ without bound ligand ${ }^{32}$. Here we present the crystal structure of the IL-15-IL-15R $\alpha$ complex.

\section{RESULTS}

\section{Structure determination}

We expressed the mature polypeptide of IL-15, as well as residues 1-102 of the processed form of IL-15R $\alpha$, each fused cleavably to thioredoxin, in Escherichia coli. Residues 1-102 of IL-15R $\alpha$ include the sushi domain (residues 1--65) and a 20-residue extension known to substantially increase the affinity of IL-15R $\alpha$ for IL-15 (ref. 33). We did not include residues 103-175, which are expected to form an extended threonine- and proline-rich sequence, in the construct that crystallized. A longer construct, consisting of residues 1-173 of the mature polypeptide, underwent substantial degradation during purification.

Two crystal forms with space groups of $\mathrm{P} 2{ }_{1} 2{ }_{1} 2$ and $\mathrm{P} 2{ }_{1} 2_{1} 2_{1}$ that diffracted to $1.85 \AA$ and $2.0 \AA$, respectively, ultimately provided ten separate views of the IL-15-IL-15R $\alpha$ complex. After unsuccessful attempts to solve the structure by molecular replacement, we used single anomalous dispersion to determine phases from a single platinum derivative of a $\mathrm{P}_{1} 2_{1} 2$ crystal. We then extended and improved phases with native data to $1.85 \AA$. We solved the $\mathrm{P} 2{ }_{1} 2_{1} 2_{1}$ form by molecular replacement. We found that the asymmetric unit of the $\mathrm{P} 2{ }_{1} 2{ }_{1} 2$ crystals contains two copies

\section{Data collection}

Resolution $(\AA)$

Cell constants $(\AA)^{\mathrm{a}}$

Space group

Measured reflections

Unique reflections

Completeness (\%)

$R_{\text {merge }}(\%)^{\mathrm{b}}$

$I / \sigma(I)$

Refinement statistics

Resolutions ( $\AA$ )

Reflections used

$R_{\text {cryst }}(\%)^{\mathrm{c}}$

$R_{\text {free }}(\%)^{\mathrm{d}}$

Completeness (\%)

Atoms

Protein; water

r.m.s. deviation from ideality

Bond lengths $(\AA)$; bond angles $\left({ }^{\circ}\right)$

B-factors

Average $B$ factors $\left(\AA^{2}\right)$

Main chain; side chain; water

B-factor r.m.s. deviations ( $\AA^{2}$ )

Main-chain bonds; side-chain bonds

Main-chain angles; side-chain angles
Native 1 30.0-1.85 (1.92-1.85)

$78.4,120.0,49.5$

$\mathrm{P} 2{ }_{1} 2{ }_{1} 2$

375,278

$39,742(3,422)$

$98.2(86.1)$

$0.069(0.380)$

$26.38(2.52)$

Native 1

1.85 (1.90-1.85)

37,703

$20.2(30.9)$

25.2 (36.7)

$100(100)$

3,$036 ; 228$

$0.018 ; 1.7$

$0.019 ; 1.8$

Native 2

116,633

100 (100)
2.00 (2.0-2.05)

20.5 (25.0)

25.0 (30.9)

11,998; 519

Values in parentheses are for the outer shell. Native 1 and Native 2 are native data sets of the P2 ${ }_{1}{ }_{1} 2$ crystal and $\mathrm{P} 2{ }_{1} 2_{1} 2_{1}$ crystal, respectively; $\mathrm{K}_{2} \mathrm{PtCL}_{6}$ is the heavy-atom derivative data set of the $\mathrm{P} 2{ }_{1}{ }_{2} 2$ crystal used for initial phase calculation.

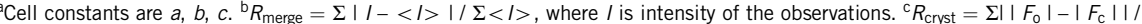
$\Sigma\left|F_{0}\right|$, where $F_{0}$ and $F_{\mathrm{c}}$ are the observed and calculated structure factors, respectively. ${ }^{\mathrm{d}} R_{\text {free }}$ is calculated as for $R_{\text {cryst }}$, but for the test set comprising reflections not used in refinement. 
a

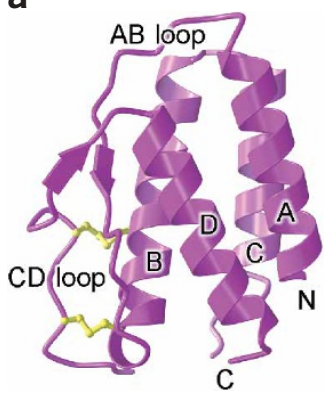

b

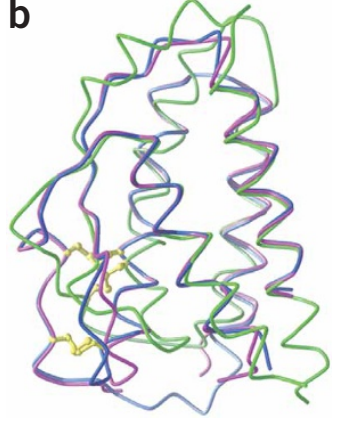

C

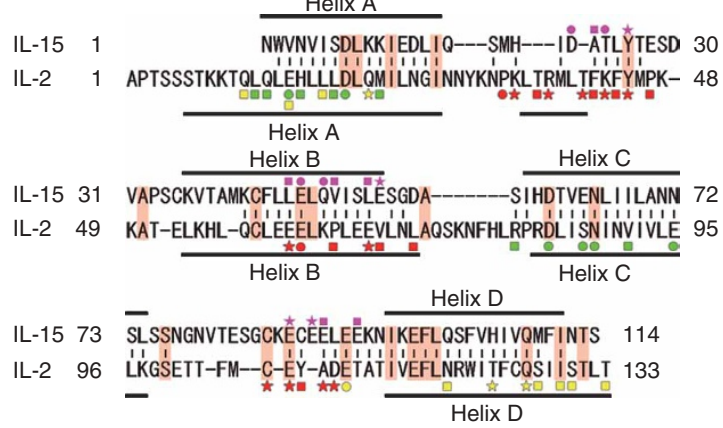

Figure 2 Views of IL-15 and sequence comparison. (a) IL-15, with the positions of two disulfide bridges in yellow. N, amino terminus; C, carboxyl terminus. (b) Superposition of the two copies of IL-15 in the P $2{ }_{1} 2{ }_{1} 2$ crystal (magenta and blue) and IL-2 (green). (c) Structure-based alignment of amino acid sequences of human IL-15 and human IL-2. Pink shading indicates residues sharing sequence identity. Symbols above and below sequences indicate residues involved in bonds: circles, hydrogen bonds; squares, van der Waals contacts; stars, both hydrogen bonds and van der Waals contacts; magenta, binding to IL-15R $\alpha$; red, binding to IL-2R $\alpha$; green, binding to IL-2R $\beta$; yellow, binding to $\gamma \mathrm{C}$. Lines between IL-15 and IL-2 sequences indicate residues with an r.m.s. deviation in the $\mathrm{C} \alpha$ position of less than $3.0 \AA$.

most similar in the regions of the $\mathrm{A}, \mathrm{C}$ and $\mathrm{D}$ helices, which form contacts with the IL-2R $\beta$ and $\gamma c$ subunits in IL-2R complexes ${ }^{30,31}$. The least-conserved regions comprise (i) the $\mathrm{AB}$ and $\mathrm{BC}$ loops, which are shorter in IL-15 by five and seven residues, respectively, (ii) the contributes to the $\alpha$-chain receptor-binding specificity.

Of the two disulfide bonds formed in IL-15, only the Cys42-Cys88 pair has been reported before for any small, four helix-bundle cytokine, and it has been reported only for IL-2 (ref. 35). Along with the shorter $\mathrm{AB}$ loop of IL-15, the disulfide bond probably stabilizes the IL-15R $\alpha$-binding surface. The ten copies of IL-15 were very similar (r.m.s. deviation of $0.53-0.75 \AA$ for $106-110$ residues), indicating that IL-15 is relatively rigid. However, the CD loop was stabilized by crystal packing in only three of the ten molecules; in one of these, the structure of the base of the $\mathrm{AB}$ loop was also altered. This localized flexibility is unlikely to affect receptor interactions.

IL-2R $\beta$ and $\gamma c$ bind orthogonally to the A and C helices of IL- 2 and the A and D helices of IL-2, respectively. These interactions bury twelve and ten residues in IL-2, respectively, three and two of which were conserved in IL-15 (Fig. 2c). In many cases, the residues that are different in IL-15 are nevertheless expected to participate in favorable interactions with IL-2R $\beta$ and $\gamma$ c. For example, Glu64 of IL-15, which is structurally equivalent to Ser87 of IL-2, may form hydrogen bonds or a salt bridge with Arg42 of IL-2R $\beta$. Similarly, Ser7 of IL-15, which is structurally equivalent to Leu19 of IL-2, may make hydrogen bonds rather than van der Waals contacts with His133 and Tyr134 of IL-2R $\beta$. There is relatively poor conservation of the $\gamma c$ subunitbinding surface for the IL-4- $\gamma \mathrm{c}$ interaction ${ }^{37}$ (Supplementary Fig. 2 online). Along with the structural similarities of the A, C and D helices of IL-15 and IL-2, which form contacts with IL-2R $\beta$ and $\gamma \mathrm{c}$, the similar physicochemical properties of these substituted amino acids in IL-15 and IL-2 suggest that the two cytokines may engage IL-2R $\beta$ and $\gamma c$ in a similar way.

\section{IL-15R $\alpha$ structure}

The sushi domain of IL-15R $\alpha$, reported before in an NMR-derived structure $^{32}$, showed conventional ABCC'DE topology, in contrast to the two strand-sharing sushi domains of IL-2R $\alpha^{29}$ (Fig. 3a). Structural comparisons using Dali showed that the sushi domain of IL-15R $\alpha$ was more similar to the amino-terminal sushi domain of decay-accelerating factor (Protein Data Bank accession code, 1ojv; r.m.s. deviation of $1.8 \AA$ for 61 residues) and the carboxy-terminal, IL-2-nonbinding sushi domain of IL-2R $\alpha$ (Protein Data Bank accession code, 1z92; r.m.s. deviation of $2.3 \AA$ for 63 residues) than to the IL-2-binding sushi domain of IL- $2 \mathrm{R} \alpha$, which was not selected at all by Dali. Nevertheless, the IL-15R $\alpha$ sushi domain and the IL-2-binding sushi domain of IL- $2 \mathrm{R} \alpha$ showed some notable similarities. Two $\beta$-turns that created bulges in the $\mathrm{CC}^{\prime}$ and $\mathrm{C}^{\prime} \mathrm{D}$ loops, as well as the C-E-strand disulfide bond of IL-2R $\alpha$, were recapitulated in IL-15R $\alpha$ (Fig. 3b,c). Moreover, a very short two-stranded $\beta$-sheet also formed between strand $C^{\prime}$ (Lys34 and Arg35) and strand $E^{\prime}$ (Cys63 and Ile64) in IL-15R $\alpha$. Together, these conserved features created a rigid base for ligand binding and stabilized, through a hydrogen bond between Gly32 and Asp66 (Fig. 3b), the carboxyl terminus of strand E, which also contacted IL-15. The carboxyl terminus of the IL-15R $\alpha$ sushi domain was extended into a short helix of five residues, whereas the carboxyl terminus of the IL-2-binding sushi domain of IL-2R $\alpha$ is a

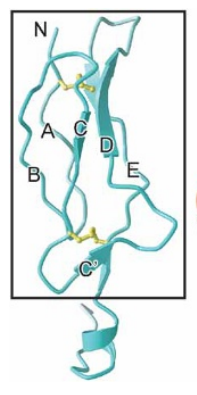

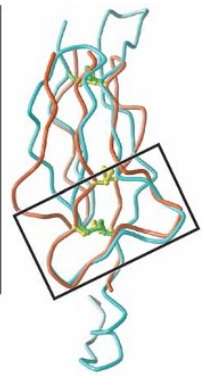

b
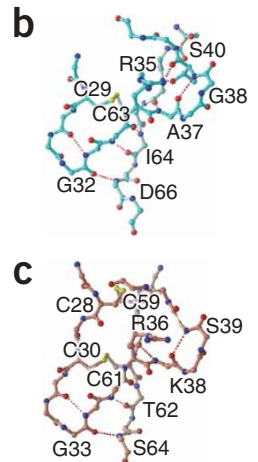

Figure 3 Views of IL-15R $\alpha$. (a) IL-15R $\alpha$ structure versus IL-2R $\alpha$ structure. Left, IL-15R $\alpha$ with the positions of two disulfide bridges in yellow; the sushi domain is outlined. $\mathrm{N}$, amino terminus. Right, superposition of the IL-2-binding domain of IL-2R $\alpha$ (red) onto IL-15R $\alpha$ (cyan); green, disulfide bonds of IL-15R $\alpha$; yellow, disulfide bonds of IL-2R $\alpha$. The CD-loop regions of IL-15R $\alpha$ and IL-2R $\alpha$ are outlined and are presented in more detail in $\mathbf{b}$ and c, respectively. (b,c) Structural comparison of the CD loops and $E$ strands of IL-15R $\alpha$ (b) and IL-2R $\alpha$ (c). Dashed lines, hydrogen bonds; yellow, sulfur atoms of cysteine residues forming disulfide bonds. 
Figure 4 Recognition of IL-15 by IL-15R $\alpha$. (a) The IL-15-IL-15R $\alpha$ complex: magenta, IL-15; cyan, IL-15R $\alpha$. The interface is divided into three regions (outlined): top, middle and bottom.

(b) Enlargement of the binding area of the three regions from a of the IL-15-IL-15R $\alpha$ interface. Dashed red lines, hydrogen bonds involved in the intermolecular association. The top region consists of a salt bridge (formed between Glu53 of IL-15 and Arg26 of IL-15R $\alpha$ ), two hydrogen bonds (between Asp22 in IL-15 and Arg26 in

음 IL-15R $\alpha$, and between Glu53 in IL-15 and Ser40 in IL-15R $\alpha$ ) and three van der Waals contacts (between Ala23 and Val49 of IL-15 and Leu42 of IL-15R $\alpha$ ). The middle region consists of a conserved salt bridge (between Arg35 of IL-15R $\alpha$ and Glu46 of IL-15), which is also found in IL-2-IL-2R $\alpha$ complexes $^{29-31}$. Tyr26 of IL-15, which is also conserved in IL-2, forms a hydrogen bond with the main-chain nitrogen of Arg35 in IL-15R $\alpha$, and its side chain forms a stacking interaction in the plane of the salt bridge. The bottom region is composed of a hydrogen bond (between Glu87 of IL-15 and His71 of IL-15R $\alpha$ ), as well as van der Waals contacts (between Glu87, Glu89 and Glu90 of IL-15, and Ile64 and Pro67 of IL-15R $\alpha$ ). (c) Binding faces, presented as GRASP surfaces of IL-15R $\alpha$ (left) and IL-15 (right). Blue, positive charge; red, negative charge (contoured at $\pm 10.0 \mathrm{kT}$ ). (d) The water-mediated hydrogen-bond network. Two stable water molecules form a hydrogen-bond network from Thr24 of IL-15 to Ser41 of IL-15R $\alpha$, filling the space between the middle of the binding site and the top of the binding site.
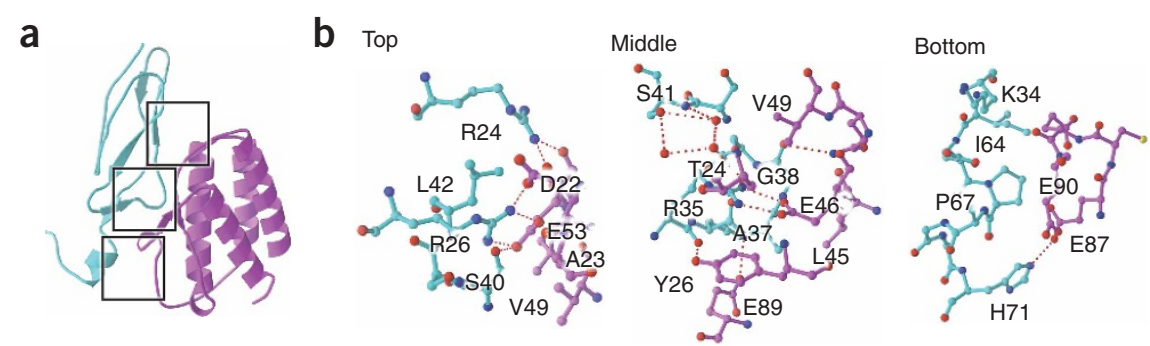

C
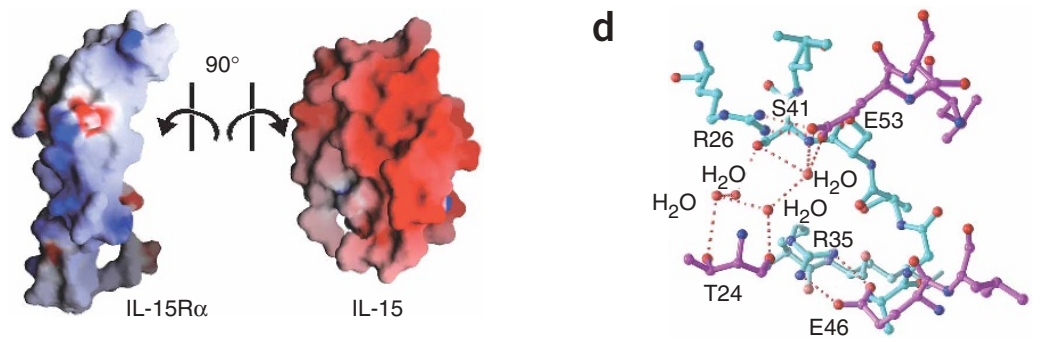

elaborated in the form of a second sushi domain, with which it exchanges strands ${ }^{29-31}$. The $C^{\prime} D$ loop and $C^{\prime} E^{\prime}$ sheet similarities of the ligand-binding IL- $15 R \alpha$ and IL- $2 \mathrm{R} \alpha$ sushi domains seem to be the most highly conserved remnants of the probable precursor of these receptors because of their central function in ligand binding.

The ten copies of IL-15R $\alpha$ in the crystals were very similar (r.m.s. deviation of $0.36-0.86 \AA$ for $71-75$ residues); in particular, the sushi domains of IL-15R $\alpha$ were almost identical (data not shown). The structure of the ligand-bound form of IL-15R $\alpha$ demonstrated by the

a

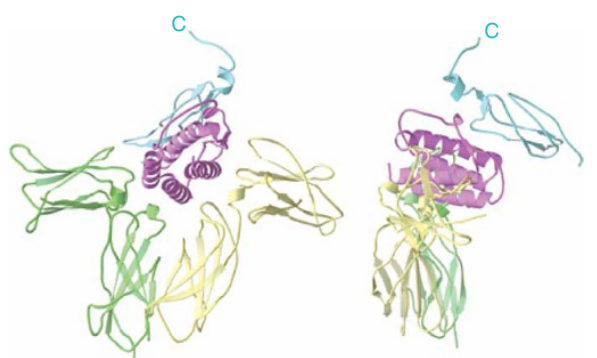

b

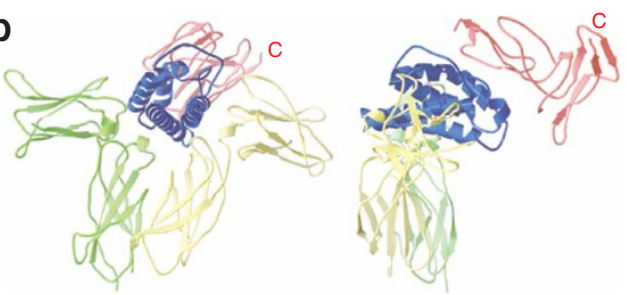

Figure 5 Comparison of a modeled IL-15 signaling complex and the quaternary IL-2 signaling complex ${ }^{30}$. Magenta, IL-15; cyan, IL-15R $\alpha$; green, IL-2R $;$; yellow, $\gamma$ c; blue, IL-2; red, IL-2R $\alpha$. (a) Two orthogonal views of the IL-15-IL-15R $\alpha$ complex modeled with IL-2R $\beta$ and $\gamma c$. (b) The quaternary IL-2 receptor complex (Protein Data Bank accession code, 2b5i). 'C', positions of the carboxyl terminus of the $\alpha$-subunit of each complex. crystal structures was similar to that of the non-ligand-bound IL-15R $\alpha$ derived by NMR analysis ${ }^{32}$ (r.m.s. deviation of $1.75 \AA$ for 66 residues). There were minor structural differences in the $\mathrm{AB}, \mathrm{BC}, \mathrm{CD}$ and $\mathrm{DE}$ loops, as well as in the E strand, that could be the result of ligand binding. It seems apparent, however, that no large-scale structural rearrangements accompany the formation of the IL-15IL-15R $\alpha$ complex.

\section{IL-15-IL-15R $\alpha$ complex structure}

In the IL-15-IL-15R $\alpha$ complex, IL-15R $\alpha$ used the carboxy-terminal half of its sushi domain to perch in a parallel orientation on the side and toward the top of IL-15 (Fig. 4a). Overall, the structure of the IL-15-IL-15R $\alpha$ complex was very similar to the complex formed by IL-2R $\alpha$ and IL-2 (r.m.s. deviation of $1.96 \AA$ for 126 residues). The binding of IL-15 to IL-15R $\alpha$ seemed to involve narrow, contiguous surfaces of about $10 \AA \times 25 \AA$ on each molecule. The interface core formed around the C'D-loop bulge of the receptor (involving Arg35, Ala37 and Gly38), which fitted into a shallow, concave surface composed of the $\mathrm{AB}$ (Thr24, Tyr26) and $\mathrm{CD}$ loops (Glu89 and, via water, Glu93), and the B helix (Glu46 and Gln48) of IL-15 (Fig. 4b). The core of the binding surface was flanked on one side by contacts between the AB loop (Asp22) and helix B (Glu53) of IL-15, and strands C (Arg24 and Arg26) and E (Ser40 and Leu42) of the receptor (Fig. $\mathbf{4 b}$ ) and on the other side by the interaction of the CD loop (Glu87 and Glu90) of IL-15 with the $\mathrm{C}^{\prime}$ strand (Lys34) and short helix (Pro67 and His71) of IL-15R $\alpha$ (Fig. 4b). However, Glu87 of IL-15 formed a hydrogen bond with His71 of IL- $15 R \alpha$ in only eight of ten complexes; in the remaining two complexes, the aliphatic portion of Glu87 formed a hydrophobic contact with Pro67 of IL-15R $\alpha$. In total, 31 residues $\left(1,214-1,224 \AA^{2}\right)$ were buried in the IL-15-IL-15R $\alpha$ complex. These residues formed seventeen van der Waals contacts, eight hydrogen bonds and two salt bridges.

Despite the considerable differences in the structures of the ligandbinding sushi domains of IL-15R $\alpha$ and IL-2R $\alpha$, the binding 


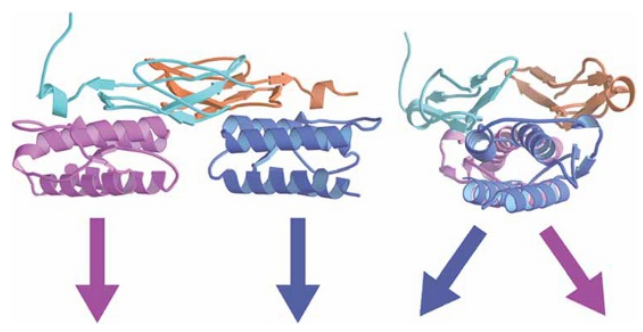

Figure 6 Two orthogonal views of the IL-15-IL-15R $\alpha$ complex dimer seen in the asymmetric unit of the $\mathrm{P} 2{ }_{1} 2{ }_{1} 2$ crystals. Arrows indicate the positions of the main axes of the IL-2R $\beta-\gamma c$ heterodimers after superposition of each IL-15 molecule in the crystallographic dimer with IL-2 from the IL-2 quaternary signaling complex (not shown) ${ }^{30}$

'footprints' of IL-15R $\alpha$ on IL-15 and IL-2R $\alpha$ on IL-2 showed substantial overlap (Supplementary Fig. 3 online). However, a much larger surface area $\left(1,686 \AA^{2} ; 41\right.$ residues) involving more contacts (31 van der Waals contacts, 11 hydrogen bonds and 1 salt bridge) was evident in the IL-2-IL-2R $\alpha$ complex. Somewhat paradoxically, the IL$15-I L-15 R \alpha$ complex is three orders of magnitude more stable than the IL-2-IL-2R $\alpha$ complex $^{10,11}$ (dissociation constant, $38 \mathrm{pM}$ versus $28.1 \mathrm{nM})$. However, the slightly concave surface of IL-15 showed better shape complementarity with the binding surface of IL- $15 \mathrm{R} \alpha$ than the equivalent surfaces in the IL-2-IL-2R $\alpha$ complex. An algorithm measuring the degree of geometric fit between two protein surfaces $^{38}$ gave scores of $0.74-0.77$ for the IL-15-IL-15R $\alpha$ complex. Those values were similar to those of constitutive oligomeric complexes and much higher than that measured for the IL-2-IL-2R $\alpha$ complex (0.659). In addition, the interaction of exclusively positively charged residues (IL-15R $\alpha$ ) or negatively charged residues (IL-15) in the IL-15-IL-15R $\alpha$ complex (Fig. 4c) resembled interactions of the RNase barnase in complex with its inhibitor barstar ${ }^{39}$, which is also noted for its high affinity. In the barnase-barstar complex, long-range electrostatic interactions generate a very high binding 'on rate 39 (over $\left.10^{9} \mathrm{M}^{-1} \mathrm{~s}^{-1}\right)$. In contrast, the 'on rate' for IL-15-IL-15R $\alpha$ binding, although high $\left(4 \times 10^{5} \mathrm{M}^{-1} \mathrm{~s}^{-1}\right)^{10}$, is not notable.

In addition to the very high degree of geometric and electrostatic complementarity noted above, water was crucial in stabilizing the IL-15-IL-15R $\alpha$ complex. Water molecules, which should have been detectable at a resolution of $2.3 \AA$, were not visible in the IL-2-IL-2R $\alpha$ binding interface ${ }^{30}$. In contrast, a particularly stable pair of hydrogenbonded water molecules (B factors of $2.0 \AA^{2}$ for both water molecules versus $20.1 \AA^{2}$ for all other water molecules) were present in the concave cleft in the core of the IL-15-IL-15R $\alpha$ interface, where they participated in a network of hydrogen bonds extending from Ser41 of IL-15R $\alpha$ to Thr24 and Glu53 of IL-15 (Fig. 4d). Ordered water molecules are also a feature of the interface of the barnase-barstar complex ${ }^{40}$. It is very likely that this network of hydrogen bonds, which also stabilized the salt bridge formed by Glu53 of IL-15 and Arg26 of IL- $15 R \alpha$, contributed substantially to the high affinity of the IL-15-IL$15 \mathrm{R} \alpha$ interaction. A second salt bridge corresponding to the only salt bridge in the IL-2 system, involving Arg35 of IL-15R $\alpha$ and Glu46 of IL-15, further stabilized the interaction. Overall, the very high affinity of the IL-15-IL-15R $\alpha$ complex is the result of combined effects of electrostatic and geometric complementarity, both of which are highly dependent on the extended network of ordered water molecules found at the interface. The concave IL-15R $\alpha$-binding surface of IL-15 has a shallow pocket that is lined with charged or polar residues (Gln48, Glu53, Glu89 and Glu93) and has two hydrophobic residues (Val49 and Tyr26) at its base (Supplementary Fig. 4 online). The physicochemical properties of this pocket are such that small molecules could be designed that might bind IL-15 with a high degree of specificity.

\section{A quaternary IL-15-IL-15R $\alpha-I L-2 R \beta-\gamma$ c complex model}

Superposition of IL-15 in the IL- $15 R \alpha$ complex with IL-2 in the complex with IL-2R $\alpha$, IL-2R $\beta$ and $\gamma c$ allowed assembly of a plausible model of a quaternary IL-15-receptor complex (Fig. 5a). The crystallized portion of IL-15R $\alpha$ is unglycosylated, whereas IL-15 has three putative $N$-glycosylation sites: Asn71, Asn79 and Asn112. Asn79 is located at the base of IL-15, some distance from the binding site of IL-15R $\alpha$ and the probable binding sites of IL-2R $\beta$ and $\gamma c$. Asn71 and Asn112, however, are located at the ends of helices $C$ and $D$, adjacent to the putative binding sites for IL-2R $\beta$ and $\gamma c$, respectively. The model suggests that formation of the quaternary IL-15-IL-15R $\alpha-$ IL$2 \mathrm{R} \beta-\gamma \mathrm{c}$ complex is unlikely to be precluded by glycosylation of Asn71 and Asn112. However, the 'on rate' for binding might be lower, an effect that would be ameliorated to some extent by the prebinding of IL-15 to IL-15R $\alpha$.

The quaternary IL-15 and IL-2-receptor complexes were very similar (Fig. 5b). Notably, the carboxyl termini of IL-15R $\alpha$ and IL-2R $\alpha$ occupied similar membrane-distal positions in terms of the membrane anchorage of IL-2R $\beta$ and $\gamma c$, consistent with the possibility that both IL-15R $\alpha$ and IL-2R $\alpha$ might present IL-15 and IL-2 in trans to cells expressing IL-2R $\beta$ and $\gamma$ c. Superimposition of the quaternary IL-15 complex on the two copies of the IL-15-IL-15R $\alpha$ complex found in the asymmetric unit of the $\mathrm{P} 2{ }_{1} 2{ }_{1} 2$ crystals showed that the main axes of IL-2R $\beta$ and $\gamma c$ are unlikely to be parallel (Fig. 6). A substantial entropic barrier therefore probably prevents the formation of such a complex.

\section{DISCUSSION}

The binding of IL-15 to IL-15R $\alpha$ is of extremely high (picomolar) affinity. However, we found here that the IL-15-IL-15R $\alpha$ interaction conspicuously lacked a binding 'hot spot' and was instead stabilized by charge-charge and van der Waals interactions resulting from a high degree of electrostatic and geometric complementarity, as well as an extensive water-mediated hydrogen-bonding network. In these ways, the IL-15-IL-15R $\alpha$ complex resembles the barnase-barstar complex ${ }^{39}$. The lower electrostatic and geometric complementarity, together with the lack of an extensive water-mediated hydrogen-bonding network in the IL-2-IL-2R $\alpha$ complex, may account for the difference in affinity of three orders of magnitude for the IL-15-IL-15R $\alpha$ and IL-2-IL-2R $\alpha$ interactions 9 .

The proinflammatory effects of IL-15 make it a very appealing therapeutic target. Val49 and Tyr26, which are surrounded by Gln48, Glu53, Glu89 and Glu93, form the interior of a relatively hydrophobic, potentially 'druggable' pocket in the concave IL-15R $\alpha$-binding surface of IL-15. However, it is difficult to envisage how any small compound could possibly compete with the extremely high-affinity interaction of IL-15 with IL-15R $\alpha$. The affinity of the complex is in fact so high that it may be functionally irreversible; very little soluble IL-15 is detectable in plasma, and receptor-bound IL-15 survives endocytosis and receptor re-expression at the cell surface ${ }^{1}$. In this sense, the IL-15-IL-15R $\alpha-$ IL-2R $\beta-\gamma c$ receptor system blurs the distinction between cell-cell recognition and the activity of soluble effectors. The requirement that individual cell-cell contacts be weak so that cellular interactions can be transient is probably satisfied by the substantially lower affinity (13.5 nM) of IL-15 for IL-2R $\beta-\gamma c$ complexes ${ }^{10}$.

Despite the low sequence homology between IL-15 and IL-2 and the distinct architecture of IL- $15 \mathrm{R} \alpha$ and IL-2R $\alpha$, the structure we have 
presented here emphasizes the evolutionarily relatedness of the IL-15IL- $15 R \alpha$ and IL-2-IL-2R $\alpha$ receptor systems. This is most evident in the manner of cytokine recognition of each of the receptors. The core of the binding interaction, involving a conserved tyrosine (Tyr26 in IL-15) and a conserved salt bridge (between Glu46 of IL-15 and Arg35 of IL-15R $\alpha$ ) is shared by both systems. Moreover, the receptor-binding 'footprints' on each cytokine showed a notable degree of overlap. Given the large differences in receptor architectures, the IL-15 and IL-2 receptor-binding systems nevertheless may be the products of very ancient gene duplications.

The asymmetric unit of each crystal form consisted of paired copies of the IL-15-IL-15R $\alpha$ complex, together forming a rod-like structure. The IL-6R complex consists of 2:2:2 ratios of the cytokine, IL-6R $\alpha$ and gp130 subunits ${ }^{41}$, raising the possibility that the IL-15-IL-15R $\alpha$ dimer might be physiologically important. However, the interface formed by the dimer-forming IL-15R $\alpha$ subunits was relatively small $\left(459 \AA^{2}\right)$ and hydrophilic. Modeling with the IL-2R $\beta$ and $\gamma c$ subunits also indicated that when bound to IL-15, the two halves of the bivalent complex of IL-2R $\beta$ and $\gamma c$ would not lie orthogonal to the membrane. Finally, we failed to detect formation of oligomers by the IL-15-IL-15R $\alpha$ complex in solution during any stage of its preparation. Thus, we conclude that the IL-15-IL-15R $\alpha$ dimer is unlikely to be physiologically important.

The observation that IL-15 is presented in trans to T cells and B cells by antigen-presenting cells provides strong support for the proposition that cytokines may generally function in the confines of cell-cell interfaces and that one function of the immunological synapse is to direct delivery of effector functions such as cytokine secretion and thereby limit bystander $\mathrm{T}$ cell activation ${ }^{42,43}$. An important issue is whether IL-15 is unique in being presented in trans in vivo. IL-2 and IL-15 form a distinct subset of the $\gamma c$-dependent family of cytokines insofar as their receptors consist of a third subunit, the $\alpha$-chain, in addition to two shared signaling subunits ${ }^{1}$. Assuming that IL-15 and IL-2 engage the shared IL-2R $\beta-\gamma c$ subunits in a similar way, which is not unlikely given the structural homology between the receptorbinding surfaces of IL-15 and IL-2, the topologies of the quaternary signaling complexes may be very similar. In particular, the carboxyl termini of IL- $15 R \alpha$ and IL-2R $\alpha$ occupy similar membrane-distal positions. Therefore, there seem to be no structural obstacles that would prevent IL-2R $\alpha$-expressing cells from presenting IL- 2 in trans to cells expressing the IL-2R $\beta-\gamma c$ complex. Moreover, the threonine- and proline-rich sequence of IL-2R $\alpha$ is probably heavily $O$-glycosylated and therefore relatively rigid, to the extent that a substantial entropic barrier might prevent such a structure from 'reaching back' to coengage IL-2R $\beta-\gamma c$ expressed on the same cell in cis. In trans interactions are unlikely to be impeded in the same way. In addition, with dimensions of about $50 \AA$ for IL-2R $\beta-\gamma c$ and $100-125 \AA$ for IL-2-IL2R $\alpha$ (calculated with a value of $2-2.5 \AA$ per residue for mucinlike sequences $\left.{ }^{44}\right)$, the size of the engaged complex matches the intermembrane distance of the immunological synapse (about $150 \AA)^{45}$. The finding that the survival of IL-15R $\alpha$-expressing T cells is not greater than that of IL-15R $\alpha$-deficient $\mathrm{T}$ cells suggests that, at least in the context of IL-15 sensitivity, no advantage results from the cis expression of all three receptor subunits ${ }^{46,47}$. It may thus be possible that like IL-15, IL-2 can be presented in trans by an IL-2-secreting $\mathrm{T}$ cell to IL-2R $\beta$ - $\gamma$ c-expressing cells with which $\mathrm{T}$ cells form immunological synapses. In this way, for example, an antigen-experienced IL-2R $\alpha^{+} \mathrm{T}$ cell might promote the IL-2-dependent proliferation of naive IL-2R $\alpha^{-}$T cells or B cells. In vitro, IL- $2 \mathrm{R} \alpha^{+}$ cell lines and transfectants are physically capable of inducing the proliferation of IL-2R $\alpha$-deficient T cells in the presence of exogenous IL-2 (ref. 48). However, the memory responses of T cells adoptively transferred into IL-2R $\alpha^{+}$hosts are compromised by IL- $2 \mathrm{R} \alpha$ deficiency ${ }^{49}$, indicating that at least some IL-2 responses are dependent on the cis expression of all three components of IL-2R. Exactly how IL-15 and IL-2 are presented may depend only on whether their respective receptor $\alpha$-chains are expressed in cis or in trans relative to $\gamma \mathrm{c}$ and IL-2R $\beta$. This may broaden the utility of IL- 2 and IL-15 by allowing responses to these cytokines to be contact dependent.

\section{METHODS}

Protein expression and purification. The recombinant extracellular domains of human IL-15R $\alpha$ (residues 1-102) and IL-15 (residues 1-114) were expressed in E. coli and were purified by nickel-nitrilotriacetic acid metal affinity resin (Qiagen). IL-15R $\alpha$ was purified by anion-exchange chromatography on a RESOURCE Q column (GE Healthcare), then was enzymatically digested with enterokinase before repurification by cation-exchange chromatography on RESOURCE S column (GE Healthcare). IL-15 was purified by cation-exchange chromatography on a RESOURCE $S$ column followed by further purification by size-exclusion chromatography on a HiLoad 16/60 Superdex 75 preparationgrade column (GE Healthcare). The binding ability and stoichiometry of IL-15 and IL-15R $\alpha$ were assessed by native gel electrophoresis. IL-15 bound stoichiometrically to IL- $15 R \alpha$. IL-15 and IL-15R $\alpha$ were mixed in a 1:1 molar ratio and the complex was purified by gel-filtration chromatography on the HiLoad $16 / 60$ Superdex 75 column. The purified complex was concentrated to $6 \mathrm{mg} / \mathrm{ml}$ for crystallization trials.

Crystallization and data collection. The TOPAZ system (Fluidigm) was used for initial crystallization screening. The IL-15-IL15R $\alpha$ complex crystals for structure analysis were then grown by the sitting-drop vapor-diffusion method by mixture of a protein solution with an equal volume of reservoir solution containing $0.15 \mathrm{mM}$ sodium citrate, $\mathrm{pH} 5.5$, and $0.85 \mathrm{M}$ sodium malonate. The IL-15-IL-15R $\alpha$ complex was crystallized into two crystal forms in the same conditions. One crystal form belonged to space group $\mathrm{P} 2{ }_{1} 2_{1} 2$ with cell dimensions of $a=78.4 \AA, b=120.0 \AA$ and $c=49.5 \AA$. The other crystal form belonged to space group $\mathrm{P} 22_{1} 2_{1} 2_{1}$ with cell dimensions of $a=81.8 \AA, b=127.0 \AA$ and $c=191.3 \AA$. Because of the failure of molecular replacement with the sushi domain in the IL-15R $\alpha$ NMR structure ${ }^{32}$ or with the structure of IL-2 or the IL-2-IL-2R $\alpha$ complex ${ }^{29-31}$, heavy metal-derivative crystals were used for phasing. $\mathrm{P} 2{ }_{1}{ }_{1} 2$ native crystals, $\mathrm{K}_{2} \mathrm{PtCl}_{6}$ derivative crystals (heavy-atom (platinum) derivative of the $\mathrm{P} 2_{1} 2_{1} 2$ crystal) and $\mathrm{P} 2_{1} 2_{1} 2_{1}$ native crystals were transferred to crystallization solution containing $10 \%(\mathrm{vol} / \mathrm{vol})$ glycerol, and data were then collected at beamline NW12A of Photon Factory Advanced Ring, with Quantum 210 (Area Detector Systems Corporation) for detection. Diffraction data were processed and scaled with the HKL program suite ${ }^{50}$ (statistics, Table 1).

Structure determination. The phases of the $\mathrm{P} 2{ }_{1} 2_{1} 2$ crystal form were initially determined to a resolution of $2.9 \AA$ with the $\mathrm{K}_{2} \mathrm{PtCl}_{6}$ derivative data by the single anomalous dispersion method with the program Phenix ${ }^{51}$. The phases were then extended to $1.85 \AA$ with $\mathrm{P} 2{ }_{1} 2_{1} 2$ native data with Resolve in Phenix. Automated model building was unsuccessful with Phenix and ARP/wARP software. The phases were further improved with the program $\mathrm{DM}^{52}$ and the structure was then manually built with the program $\operatorname{Coot}^{53}$ then refined with the crystallography and nuclear magnetic resonance system ${ }^{54}$ and REFMAC ${ }^{55}$ for the final stages. The $\mathrm{P} 2{ }_{1} 2_{1} 2$ crystal contained two IL-15-IL-15R $\alpha$ complexes in the asymmetric unit. The $\mathrm{P} 22_{1} 2_{1} 2_{1}$ structure was solved by molecular replacement at a resolution of $2.5 \AA$ with program $\mathrm{COMO}^{56}$ with the refined $\mathrm{P} 2{ }_{1} 2{ }_{1} 2$ crystal structure. This crystal contained eight copies of IL-15-IL-15R $\alpha$ complexes in the asymmetric unit and was refined to a resolution of $2.0 \AA$ with the crystallography and nuclear magnetic resonance system and REFMAC.

Accession codes. Protein Data Bank (coordinates of human IL-15-IL-15R $\alpha$ ): $\mathrm{P} 2{ }_{1} 2_{1} 2$ crystal, 2Z3Q; P2 ${ }_{1} 2_{1} 2_{1}$ crystal, 2Z3R.

Note: Supplementary information is available on the Nature Immunology website.

\section{ACKNOWLEDGMENTS}

Supported by a Grant-in-Aid for Creative Scientific Research from the Ministry of Education, Culture, Sports, Science and Technology, the Uehara Memorial 
Foundation, the Natio Foundation, the Astellas Foundation for Research on Metabolic Disorders, the Takeda Science Foundation, the Suzuken Memorial Foundation, the Kanae Foundation for the Promotion of Medical Science, the Astellas Foundation for Research on Medicinal Resources, Japan Research Foundation for Clinical Pharmacology, and The Wellcome Trust.

\section{AUTHOR CONTRIBUTIONS}

M.C., C.H., T.N., S.T., T.S., H.K. and Y.Y. did the research; S.J.D. and S.I. designed experiments, interpreted data and prepared the manuscript; and S.I. conceived and supervised the project.

\section{COMPETING INTERESTS STATEMENT}

The authors declare no competing financial interests.

Published online at http://www.nature.com/natureimmunology

Reprints and permissions information is available online at http://npg.nature.com/ reprintsandpermissions

1. Waldmann, T.A. The biology of interleukin-2 and interleukin-15: implications for cancer therapy and vaccine design. Nat. Rev. Immunol. 6, 595-601 (2006).

2. Alves, N.L., Arosa, F.A. \& van Lier, R.A. Common gamma chain cytokines: dissidence in the details. Immunol. Lett. 108, 113-120 (2007).

3. Surh, C.D., Boyman, O., Purton, J.F. \& Sprent, J. Homeostasis of memory T cells. Immunol. Rev. 211, 154-163 (2006)

4. Ma, A., Koka, R. \& Burkett, P. Diverse functions of IL-2, IL-15, and IL-7 in lymphoid homeostasis. Annu. Rev. Immunol. 24, 657-679 (2006).

5. Burton, J.D. et al. A lymphokine, provisionally designated interleukin T and produced by a human adult T-cell leukemia line, stimulates T-cell proliferation and the induction of lymphokine-activated killer cells. Proc. Natl. Acad. Sci. USA 91, 4935-4939 (1994).

6. Grabstein, K.H. et al. Cloning of a T cell growth factor that interacts with the $\beta$ chain of the interleukin-2 receptor. Science 264, 965-968 (1994).

7. Pettit, D.K. et al. Structure-function studies of interleukin 15 using site-specific mutagenesis, polyethylene glycol conjugation, and homology modeling. J. Biol. Chem. 272, 2312-2318 (1997)

8. Giri, J.G. et al. Utilization of the $\beta$ and $\gamma$ chains of the IL-2 receptor by the novel cytokine IL-15. EMBO J. 13, 2822-2830 (1994).

9. Giri, J.G. et al. Identification and cloning of a novel IL-15 binding protein that is structurally related to the $\alpha$ chain of the IL-2 receptor. EMBO J. 14, 3654-3663 (1995).

10. Mortier, E. et al. Soluble interleukin-15 receptor $\alpha($ IL-15R $\alpha)$-sushi as a selective and potent agonist of IL-15 action through IL-15R $\beta / \gamma$. Hyperagonist IL-15-IL-15R $\alpha$ fusion proteins. J. Biol. Chem. 281, 1612-1619 (2006).

11. Liparoto, S.F. et al. Analysis of the role of the interleukin-2 receptor $\gamma$ chain in ligand binding. Biochemistry 41, 2543-2551 (2002)

12. Anderson, D.M. et al. Functional characterization of the human interleukin- 15 receptor $\alpha$ chain and close linkage of IL15RA and IL2RA genes. J. Biol. Chem. 270, 29862-29869 (1995)

13. Budagian, V., Bulanova, E., Paus, R. \& Bulfone-Paus, S. IL-15/IL-15 receptor biology: a guided tour through an expanding universe. Cytokine Growth Factor Rev. 17, 259-280 (2006)

14. Bamford, R.N., Battiata, A.P., Burton, J.D., Sharma, H. \& Waldmann, T.A. Interleukin (IL) 15/IL-T production by the adult T-cell leukemia cell line HuT-102 is associated with a human T-cell lymphotrophic virus type I region /IL-15 fusion message that lacks many upstream AUGs that normally attenuates IL-15 mRNA translation. Proc. Natl. Acad. Sci. USA 93, 2897-2902 (1996).

15. Burkett, P.R. et al. Coordinate expression and trans presentation of interleukin (IL)$15 R \alpha$ and IL-15 supports natural killer cell and memory $\mathrm{CD} 8^{+} \mathrm{T}$ cell homeostasis. J. Exp. Med. 200, 825-834 (2004).

16. Dubois, S., Mariner, J., Waldmann, T.A. \& Tagaya, Y IL-15R $\alpha$ recycles and presents IL-15 In trans to neighboring cells. Immunity 17, 537-547 (2002).

17. Lodolce, J.P. et al. T cell-independent Interleukin $15 R \alpha$ signals are required for bystander proliferation. J. Exp. Med. 194, 1187-1194 (2001).

18. Ku, C.C. et al. Control of homeostasis of $\mathrm{CD}^{+}$memory T cells by opposing cytokines. Science 288, 675-678 (2000)

19. Thornton, A.M. Piccirillo, C.A. \& Shevach, E.M. Activation requirements for the induction of $\mathrm{CD} 4{ }^{+} \mathrm{CD} 25^{+} \mathrm{T}$ cell suppressor function. Eur. J. Immunol. 34, 366-376 (2004).

20. Lenardo, M.J. Interleukin-2 programs mouse $\alpha \beta$ T Iymphocytes for apoptosis. Nature 353, 858-861 (1991)

21. Bulfone-Paus, S. et al. Interleukin-15 protects from lethal apoptosis in vivo. Nat. Med. 3, 1124-1128 (1997)

22. Sadlack, B. et al. Ulcerative colitislike disease in mice with a disrupted interleukin-2 gene. Cell 75, 253-261 (1993).

23. Willerford, D.M. et al. Interleukin-2 receptor $\alpha$ chain regulates the size and content of the peripheral lymphoid compartment. Immunity 3, 521-530 (1995).
24. Suzuki, H. et al. Deregulated T cell activation and autoimmunity in mice lacking interleukin-2 receptor $\beta$. Science 268, 1472-1476 (1995).

25. Lodolce, J.P. et al. IL-15 receptor maintains lymphoid homeostasis by supporting lymphocyte homing and proliferation. Immunity 9, 669-676 (1998).

26. Kennedy, M.K. et al. Reversible defects in natural killer and memory CD8 T cell lineages in interleukin 15-deficient mice. J. Exp. Med. 191, 771-780 (2000).

27. Kobayashi, H. et al. Role of trans-cellular IL-15 presentation in the activation of NK cell-mediated killing, which leads to enhanced tumor immunosurveillance. Blood 105 , 721-727 (2005).

28. Oh, S. et al. Coadministration of HIV vaccine vectors with vaccinia viruses expressing IL-15 but not IL-2 induces long-lasting cellular immunity. Proc. Natl. Acad. Sci. USA 100, 3392-3397 (2003).

29. Rickert, M. et al. The structure of interleukin-2 complexed with its $\alpha$ receptor. Science 308, 1477-1480 (2005)

30. Wang, X., Rickert, M. \& Garcia, K.C. Structure of the quaternary complex of interleukin2 with its $\alpha, \beta$, and $\gamma$ c receptors. Science 310, 1159-1163 (2005).

31. Stauber, D.J. et al. Crystal structure of the IL-2 signaling complex: paradigm for a heterotrimeric cytokine receptor. Proc. Natl. Acad. Sci. USA 103, 2788-2793 (2006).

32. Lorenzen, I., Dingley, A.J., Jacques, Y. \& Grotzinger, J. The structure of the interleukin$15 \alpha$ receptor and its implications for ligand binding. J. Biol. Chem. 281, 6642-6647 (2006).

33. Wei, X.Q. et al. The Sushi domain of soluble IL-15 receptor $\alpha$ is essential for binding IL-15 and inhibiting inflammatory and allogenic responses in vitro and in vivo. J. Immunol. 167, 277-282 (2001).

34. Laskowski, R.A., MacArthur, M.W., Moss, D.S. \& Thornton, J.M. PROCHECK: a program to check the stereochemical quality of protein structures. J. Appl. Crystallogr. 26, 283-291 (1993)

35. Rozwarski, D.A. et al. Structural comparisons among the short-chain helical cytokines. Structure 2, 159-173 (1994)

36. Holm, L. \& Sander, C. Dali: a network tool for protein structure comparison. Trends Biochem. Sci. 20, 478-480 (1995).

37. Zhang, J.K., Buehner, M. \& Sebald, W. Functional epitope of common $\gamma$ chain for interleukin-4 binding. Eur. J. Biochem. 269, 1490-1499 (2002).

38. Lawrence, M.C. \& Colman, P.M. Shape complementarity at protein/protein interfaces. J. Mol. Biol. 234, 946-950 (1993).

39. Schreiber, G. \& Fersht, A.R. Rapid, electrostatically assisted association of proteins. Nat. Struct. Biol. 3, 427-431 (1996).

40. Buckle, A.M., Schreiber, G. \& Fersht, A.R. Protein-protein recognition: crystal structural analysis of a barnase-barstar complex at 2.0-A resolution. Biochemistry 33 , 8878-8889 (1994).

41. Boulanger, M.J., Chow, D.C., Brevnova, E.E. \& Garcia, K.C. Hexameric structure and assembly of the interleukin-6/IL-6 $\alpha$-receptor/gp130 complex. Science 300 2101-2104 (2003).

42. Davis, S.J. \& van der Merwe, P.A. The immunological synapse: required for T cell receptor signalling or directing T cell effector function? Curr. Biol. 11, R289-R291 (2001).

43. van Der Merwe, P.A. \& Davis, S.J. The immunological synapse-a multitasking system. Science 295, 1479-1480 (2002)

44. Jentoft, N. Why are proteins 0-glycosylated? Trends Biochem. Sci. 15, 291-294 (1990).

45. van der Merwe, P.A., Davis, S.J., Shaw, A.S. \& Dustin, M.L. Cytoskeletal polarization and redistribution of cell-surface molecules during $T$ cell antigen recognition. Semin. Immunol. 12, 5-21 (2000).

46. Burkett, P.R. et al. IL-15R $\alpha$ expression on $\mathrm{CD}^{+} \mathrm{T}$ cells is dispensable for $\mathrm{T}$ cell memory. Proc. Natl. Acad. Sci. USA 100, 4724-4729 (2003).

47. Schluns, K.S., Klonowski, K.D. \& Lefrancois, L. Transregulation of memory CD8 proliferation by IL-15R $\alpha^{+}$bone marrow-derived cells. Blood 103, 988-994 (2004).

48. Eicher, D.M. \& Waldmann, T.A. IL-2R $\alpha$ on one cell can present IL-2 to IL-2Rß/ $/$ c on another cell to augment IL-2 signaling. J. Immunol. 161, 5430-5437 (1998).

49. Williams, M.A., Tyznik, A.J. \& Bevan, M.J. Interleukin-2 signals during priming are required for secondary expansion of $\mathrm{CD}^{+}$memory T cells. Nature 441, 890-893 (2006).

50. Otwinowski, Z. \& Minor, W. Processing of X-ray diffraction data collected in oscillation mode. Methods Enzymol. 276, 307-326 (1997).

51. Adams, P.D. et al. PHENIX: building new software for automated crystallographic structure determination. Acta Crystallogr. D Biol. Crystallogr. 58, 1948-1954 (2002).

52. Cowtan, K. DM: An automated procedure for phase improvement by density modification. Joint CCP4 ESF-EACBM Newsl. Protein Crystallogr. 31, 34-38 (1994).

53. Emsley, P. \& Cowtan, K. Coot: model-building tools for molecular graphics. Acta Crystallogr. D Biol. Crystallogr. 60, 2126-2132 (2004).

54. Brunger, A.T. et al. Crystallography \& NMR system: A new software suite for macromolecular structure determination. Acta Crystallogr. D Biol. Crystallogr. 54, 905-921 (1998).

55. Winn, M.D., Murshudov, G.N. \& Papiz., M.Z. Macromolecular TLS refinement in REFMAC at moderate resolutions. Methods Enzymol. 374, 300-321 (2003).

56. Jogl, G., Tao, X., Xu, Y. \& Tong, L. COMO: a program for combined molecular replacement. Acta Crystallogr. D Biol. Crystallogr. 57, 1127-1134 (2001). 